\title{
On an inequality between pseudorandom measures of lattices
}

\author{
Katalin Gyarmati \\ Eötvös Loránd University \\ Department of Algebra and Number Theory \\ H-1117 Budapest, Pázmány Péter sétány 1/C \\ E-mail: gykati@cs.elte.hu \\ Richárd Sebók \\ Eötvös Loránd University \\ Department of Algebra and Number Theory \\ H-1117 Budapest, Pázmány Péter sétány 1/C \\ E-mail: sebokrichard.hun@gmail.com
}

\begin{abstract}
Mauduit and Sárközy proved the following inequality between the well-distribution measure and the correlation measure of order 2 : $W\left(E_{N}\right) \leq 3 \sqrt{N C_{2}\left(E_{N}\right)}$. This result has been generalized to inequalities between the combined pseudorandom measures and correlation measures of even order by the authors of the present paper. Here
\end{abstract}

Supported by National Research Development and Innovation Office, NKFIH KKP133819 and K119528.

2010 Mathematics Subject Classification: Primary 11K45

Key words and phrases: binary sequences, pseudorandomness, well-distribution, correlation. 
the multidimensional case is studied, and this inequality is extended further to the case of binary lattices.

\section{Introduction}

In 1997 Mauduit and Sárközy [8] introduced new pseudorandom measures of finite binary sequences in order to study the pseudorandom properties of these sequences. These pseudorandom measures are the following: For a binary sequence $E_{N}=\left(e_{1}, \ldots, e_{N}\right) \in\{-1,+1\}^{N}$ of length $N$, the welldistribution measure of $E_{N}$ is defined as

$$
W\left(E_{n}\right)=\max _{a, b, t}\left|U\left(E_{N}, t, a, b\right)\right|=\max _{a, b, t}\left|\sum_{j=0}^{t-1} e_{a+j b}\right|,
$$

where the maximum is taken over all $a \in \mathbb{Z}, b, t \in \mathbb{N}$ such that $1 \leq a \leq$ $a+b(t-1) \leq N$.

The correlation measure of order $k$ of $E_{N}$ is defined as

$$
C_{k}\left(E_{N}\right)=\max _{M, D}\left|V\left(E_{N}, M, D\right)\right|=\max _{M, D}\left|\sum_{n=1}^{M} e_{n+d_{1}} e_{n+d_{2}} \ldots e_{n+d_{k}}\right|,
$$

where the maximum is taken over all $D=\left(d_{1}, \ldots, d_{k}\right)$ with non-negative integers $d_{1}<\cdots<d_{k}$ and $M \in \mathbb{N}$ such that $M+d_{k} \leq N$.

Mauduit and Sárközy [8] showed that a finite binary sequence can be considered as a good pseudorandom sequence if both the well-distribution measure and the correlation measures are small. For more details see e.g., the survey paper [4].

The combined (well-distribution-correlation) pseudorandom measure of order $k$ of $E_{N}$ is defined as

$$
\begin{aligned}
Q_{k}\left(E_{N}\right) & =\max _{a, b, t, D}|Z(a, b, t, D)| \\
& =\max _{a, b, t, D}\left|\sum_{j=0}^{t-1} e_{a+j b+d_{1}} e_{a+j b+d_{2}} \ldots e_{a+j b+d_{k}}\right|,
\end{aligned}
$$


where the maximum is taken over all $a, b, t, D=\left(d_{1}, d_{2}, \ldots, d_{k}\right)$ such that all the subscripts $a+j b+d_{\ell}$ belong to $\{1, \ldots, N\}$.

In [9] Mauduit and Sárközy proved a sharp inequality between the welldistribution measure of $E_{N}$ and the correlation measure of order 2 of $E_{N}$ for every $E_{N} \in\{-1,+1\}^{N}$.

Theorem A (Mauduit and Sárközy). For $N \geq 1$ and $E_{N}=\left(e_{1}, \ldots, e_{N}\right) \in$ $\{-1,+1\}^{N}$ we have

$$
W\left(E_{N}\right) \leq 3 \sqrt{N C_{2}\left(E_{N}\right)} .
$$

Later in [3] the first author of the present paper generalized Theorem A to a similar inequality between $W$ and $C_{2 k}$. In 2015 the second author of the present paper generalized further this inequality, namely he proved the following result:

Theorem B (Sebők). For $N \geq 1$ and $E_{N}=\left(e_{1}, \ldots, e_{N}\right) \in\{-1,+1\}^{N}$, $k \in \mathbb{N}$ and for $1 \leq l \leq k$ we have

$$
Q_{k}\left(E_{N}\right) \leq 2 \sqrt{N \max _{1 \leq l \leq k} C_{2 l}\left(E_{N}\right)}
$$

Note that an important consequence of Theorems A and B is that if one needs only nontrivial upper bounds for the measures $W$ and $Q_{k}$ (but one does not need possibly sharp upper bounds), then this sort of bounds can be obtained by just estimating $C_{2 k}$ (for $k$ not very large), thus the computation can be shortened considerably; besides, it often occurs that one can find estimates in the literature for the corresponding "complete correlation" (see the references in [10] for complete correlation estimates in both one dimensional and multidimensional cases) and there is standard techniques to deduce the "incomplete" correlation estimates used in the study of pseudorandom measures from the complete ones, which may reduce the computation further.

In [9] Mauduit and Sárközy also showed that their upper bound for $W\left(E_{N}\right)$ in terms of $C_{2}\left(E_{N}\right)$ is sharp, namely in the range

$$
N^{3 / 4}(\log N)^{1 / 4} \ll W\left(E_{N}\right) \leq N
$$

(1.1) is best possible apart from a constant factor: 
Theorem C (Mauduit and Sárközy). If $m, N \in \mathbb{N}, N>N_{0}$ and

$$
N^{3 / 4} \ll m \leq N
$$

then there is a sequence $E_{N} \in\{-1,+1\}^{N}$ with

$$
W\left(E_{N}\right) \geq m
$$

and

$$
C_{2}\left(E_{N}\right) \leq 120 \max \left\{\frac{m^{2}}{N},(N \log N)^{1 / 2}\right\} .
$$

Note that it follows from (1.3) that if $m \geq N^{3 / 4}(\log N)^{1 / 4}$ then

$$
m \leq W\left(E_{N}\right) \leq 3\left(N C_{2}\left(E_{N}\right)\right)^{1 / 2}<33 m
$$

so that, indeed, the lower and upper bounds coincide apart from a constant factor.

The generalization of inequality (1.2) to multidimensional binary lattices is especially important. For lattices, the most frequently studied measures are the $Q_{k}$ 's, however, sometimes we may only have a good estimate for the correlation measures. One might like to generalize this inequality for lattices. First, we will present the definitions of multidimensional measures. The study of the multidimensional case was started by the work of Hubert, Mauduit and Sárközy. In [7] they introduced the following definitions.

Denote by $I_{N}^{n}$ the set of $n$-dimensional vectors whose coordinates are integers between 0 and $N-1$ :

$$
I_{N}^{n}=\left\{\mathbf{x}=\left(x_{1}, \ldots x_{n}\right): x_{i} \in\{0,1, \ldots, N-1\}\right\} .
$$

This set is called an $n$-dimensional $N$-lattice or briefly an $N$-lattice. Hubert, Mauduit and Sárközy [7] extended the definition of binary sequences to more dimensions by considering functions of type

$$
\eta(\mathbf{x}): I_{N}^{n} \rightarrow\{-1,+1\}
$$

called binary lattices. 
If $\mathbf{x}=\left(x_{1}, \ldots, x_{n}\right)$ so that $\eta(\mathbf{x})=\eta\left(\left(x_{1}, \ldots, x_{n}\right)\right)$ then we will simplify the notation by writing $\eta(\mathbf{x})=\eta\left(x_{1}, \ldots, x_{n}\right)$.

Let $\mathbf{u}_{\mathbf{1}}, \mathbf{u}_{\mathbf{2}}, \ldots, \mathbf{u}_{\mathbf{n}}$ be $n$ linearly independent $n$-dimensional vectors over the field of the real numbers such that the $i$-th coordinate of $\mathbf{u}_{\mathbf{i}}$ is a positive integer and the other coordinates of $\mathbf{u}_{\mathbf{i}}$ are 0 , so that, writing $z_{i}=\left|\mathbf{u}_{\mathbf{i}}\right|, \mathbf{u}_{\mathbf{i}}$ is of the form $\left(0, \ldots, 0, z_{i}, 0, \ldots, 0\right)$ with $z_{i} \in \mathbb{N}$. Let $t_{1}, t_{2}, \ldots, t_{n}$ be integers with $0 \leq t_{1}, t_{2}, \ldots, t_{n}<N$. Then we call the set

$$
B_{N}^{n}=\left\{\mathbf{x}=x_{1} \mathbf{u}_{\mathbf{1}}+\cdots+x_{n} \mathbf{u}_{\mathbf{n}}: 0 \leq x_{i} z_{i} \leq t_{i}(<N) \text { for } i=1, \ldots, n\right\}
$$

$n$-dimensional box $N$-lattice or briefly a box $N$-lattice.

Hubert, Mauduit and Sárközy [7] introduced the following measures of pseudorandomness of binary lattices (here we present the definitions in a slightly modified form as in [6] but equivalent with the ones in [7]). Let $\eta$ be a binary lattice

$$
\eta(\mathbf{x}): I_{N}^{n} \rightarrow\{-1,+1\} .
$$

Define the combined pseudorandom measure of order $k$ of $\eta$ by

$$
Q_{k}(\eta)=\max _{B, \mathbf{d}_{\mathbf{1}}, \mathbf{d}_{\mathbf{2}}, \ldots, \mathbf{d}_{\mathbf{k}}}\left|\sum_{\mathbf{x} \in B} \eta\left(\mathbf{x}+\mathbf{d}_{\mathbf{1}}\right) \ldots \eta\left(\mathbf{x}+\mathbf{d}_{\mathbf{k}}\right)\right|,
$$

where the maximum is taken over all distinct $\mathbf{d}_{\mathbf{1}}, \ldots, \mathbf{d}_{\mathbf{k}} \in I_{N}^{n}$ and all box lattices $B$ such that $B+\mathbf{d}_{\mathbf{1}}, \ldots, B+\mathbf{d}_{\mathbf{k}} \subseteq I_{N}^{n}$.

The combined measures of binary lattices are natural extensions of the combined measures of binary sequences. In certain applications one may also need the extension of the correlation measures for the multidimensional theory. These new measures were introduced by Gyarmati, Mauduit and Sárközy [5]. They introduced the following measure of pseudorandomness of binary lattices: the correlation measure of order $l$ of the lattice $\eta: I_{N}^{n} \rightarrow$ $\{-1,+1\}$ is defined by

$$
C_{\ell}(\eta)=\max _{B^{\prime}, \mathbf{d}_{1}, \ldots, \mathbf{d}_{\ell}}\left|\sum_{\mathbf{x} \in B^{\prime}} \eta\left(\mathbf{x}+\mathbf{d}_{\mathbf{1}}\right) \ldots \eta\left(\mathbf{x}+\mathbf{d}_{\ell}\right)\right|,
$$


where the maximum is taken over all distinct $\mathbf{d}_{\mathbf{1}}, \ldots, \mathbf{d}_{\ell} \in I_{N}^{n}$ and all box lattices $B^{\prime}$ of the special form

$$
B^{\prime}=\left\{\mathbf{x}=\left(x_{1}, \ldots, x_{n}\right): 0 \leq x_{1} \leq t_{1}(<N), \ldots, 0 \leq x_{n} \leq t_{n}(<N)\right\}
$$

such that $B^{\prime}+\mathbf{d}_{1}, \ldots, B^{\prime}+\mathbf{d}_{\ell} \subseteq I_{N}^{n}$.

In this paper, we will generalize Theorem $\mathrm{B}$ to $n$ dimension.

Theorem 1. For $1 \leq k, n, N \in \mathbb{N}$ and binary lattice $\eta: I_{N}^{n} \rightarrow\{-1,+1\}$ we have

$$
Q_{k}(\eta) \leq \sqrt{\left(2^{n}+k^{2}\right) N^{n} C_{2 k}(\eta)}
$$

As in the case of sequences this result shows that in order to get a "good" (but not necessary optimal) upper bound for the combined measure it is enough to estimate the correlation measures.

We will also show that Theorem 1 is sharp, namely we will prove the following result:

Theorem 2. For $1 \leq k, n \in \mathbb{N}$ and $3 / 4<c \leq 1$ there are infinitely many $N \in \mathbb{N}$ such that there exists a binary lattice $\eta: I_{N}^{n} \rightarrow\{-1,+1\}$ for which

$$
N^{c n} \gg Q_{k}(\eta) \gg \sqrt{N^{n} C_{2 k}(\eta)} \gg N^{c n},
$$

where the implied constant factors depend only on $n$ and $k$. (Here $\gg$ is Vinogradov's notation so that e.g. $f(N) \gg g(N)$ means that there is a positive constant $C$ such that for all $N$ we have $|f(N)| \geq C|g(N)|$.)

\section{Proof of Theorem 1}

We will prove that for every box lattice $B$ we have

$$
\left|\sum_{\mathbf{x} \in B} \eta\left(\mathbf{x}+\mathbf{d}_{\mathbf{1}}\right) \cdots \eta\left(\mathbf{x}+\mathbf{d}_{\mathbf{k}}\right)\right| \leq \sqrt{\left(2^{n}+k^{2}\right) N^{n} C_{2 k}(\eta)},
$$

in other words, we will prove

$$
\left|\sum_{\mathbf{x} \in B} \eta\left(\mathbf{x}+\mathbf{d}_{\mathbf{1}}\right) \cdots \eta\left(\mathbf{x}+\mathbf{d}_{\mathbf{k}}\right)\right|^{2} \leq\left(2^{n}+k^{2}\right) N^{n} C_{2 k}(\eta),
$$


from which the theorem follows.

Let

$$
B=\left\{\mathbf{x}=x_{1} \mathbf{u}_{\mathbf{1}}+\cdots+x_{n} \mathbf{u}_{\mathbf{n}}: 0 \leq x_{i} z_{i} \leq t_{i}(<N) \text { for } i=1, \ldots, n\right\}
$$

be a fixed box lattice. If $\mathbf{x} \notin I_{N}^{n}$, then we define $\eta(\mathbf{x})=0$. Now we define the boxes $A$ and $C$ as

$$
A=\left\{\mathbf{x} \in I_{N}^{n}: 0 \leq x_{i}<z_{i}(<N) \text { for } i=1, \ldots, n\right\}
$$

and

$$
C=\left\{\mathbf{x}=x_{1} \mathbf{u}_{\mathbf{1}}+\cdots+x_{n} \mathbf{u}_{\mathbf{n}}:-t_{i} \leq x_{i} z_{i} \leq t_{i}(<N) \text { for } i=1, \ldots, n\right\}
$$

Then

$$
|C|<2^{n}|B| \leq 2^{n} N^{n}
$$

We will use the notation of addition and subtraction of box lattices as the usual set addition and subtraction, namely $B_{1}+B_{2}=\left\{\mathbf{b}_{\mathbf{1}}+\mathbf{b}_{\mathbf{2}}: \mathbf{b}_{\mathbf{1}} \in\right.$ $\left.B_{1}, \mathbf{b}_{\mathbf{2}} \in B_{2}\right\}$ and $B_{1}-B_{2}=\left\{\mathbf{b}_{1}-\mathbf{b}_{\mathbf{2}}: \mathbf{b}_{\mathbf{1}} \in B_{1}, \mathbf{b}_{\mathbf{2}} \in B_{2}\right\}$. Note that

$$
C=B-B
$$

for the box lattices $B$ and $C$ defined above. It is also possible to consider the difference of a box lattice $B$ and a vector $\mathbf{x}$ :

$$
B-\mathbf{x}=\{\mathbf{b}-\mathbf{x}: \mathbf{b} \in B\} .
$$

Consider the sum

$$
S=\sum_{\mathbf{m} \in A}\left(\sum_{\mathbf{x} \in B} \eta\left(\mathbf{x}+\mathbf{d}_{\mathbf{1}}+\mathbf{m}\right) \ldots \eta\left(\mathbf{x}+\mathbf{d}_{\mathbf{k}}+\mathbf{m}\right)\right)^{2} .
$$

It is clear that

$$
\left|\sum_{\mathbf{x} \in B} \eta\left(\mathbf{x}+\mathbf{d}_{1}\right) \cdots \eta\left(\mathbf{x}+\mathbf{d}_{k}\right)\right|^{2} \leq S
$$


thus in order to prove the theorem we need to prove that

$$
S \leq\left(2^{n}+k^{2}\right) N^{n} C_{2 k}(\eta) .
$$

Clearly,

$$
\begin{aligned}
S= & \sum_{\mathbf{m} \in A}\left(\sum_{\mathbf{x} \in B} \eta\left(\mathbf{x}+\mathbf{d}_{\mathbf{1}}+\mathbf{m}\right) \ldots \eta\left(\mathbf{x}+\mathbf{d}_{\mathbf{k}}+\mathbf{m}\right)\right)^{2} \\
= & \sum_{\mathbf{m} \in A}\left(\sum_{\mathbf{x} \in B} \prod_{i=1}^{k} \eta\left(\mathbf{x}+\mathbf{d}_{\mathbf{i}}+\mathbf{m}\right)\right)\left(\sum_{\mathbf{y} \in B} \prod_{j=1}^{k} \eta\left(\mathbf{y}+\mathbf{d}_{\mathbf{j}}+\mathbf{m}\right)\right) \\
& =\sum_{\mathbf{m} \in A} \sum_{\mathbf{x}, \mathbf{y} \in B} \prod_{i=1}^{k} \eta\left(\mathbf{x}+\mathbf{d}_{i}+\mathbf{m}\right) \prod_{j=1}^{k} \eta\left(\mathbf{y}+\mathbf{d}_{j}+\mathbf{m}\right) \\
= & \sum_{\mathbf{m} \in A}\left(\sum_{\substack{\mathbf{x}, \mathbf{y} \\
\mathbf{x}, \mathbf{y} \in B}} \prod_{i=1}^{k} \eta\left(\mathbf{x}+\mathbf{d}_{\mathbf{i}}+\mathbf{m}\right) \eta\left(\mathbf{y}+\mathbf{d}_{\mathbf{i}}+\mathbf{m}\right)\right)
\end{aligned}
$$

Then

$$
\begin{aligned}
S= & \sum_{\mathbf{m} \in A} \sum_{\mathbf{x} \in B}\left(\prod_{i=1}^{k} \eta\left(\mathbf{x}+\mathbf{d}_{\mathbf{i}}+\mathbf{m}\right)\right)^{2}+ \\
& +\sum_{\mathbf{m} \in A} \sum_{\mathbf{x} \in B} \sum_{\substack{\mathbf{c} \in B-\mathbf{x} \\
\mathbf{c} \neq \mathbf{0}}} \prod_{i=1}^{k} \eta\left(\mathbf{x}+\mathbf{d}_{\mathbf{i}}+\mathbf{m}\right) \eta\left(\mathbf{x}+\mathbf{c}+\mathbf{d}_{\mathbf{i}}+\mathbf{m}\right) \\
= & \sum_{\mathbf{m} \in A} \sum_{\mathbf{x} \in B}\left(\prod_{i=1}^{k} \eta\left(\mathbf{x}+\mathbf{d}_{\mathbf{i}}+\mathbf{m}\right)\right)^{2}+ \\
& +\sum_{\substack{\mathbf{c} \in C \\
\mathbf{c} \neq \mathbf{0}}} \sum_{\mathbf{x} \in B \cap(B-\mathbf{c})} \sum_{\mathbf{m} \in A} \prod_{i=1}^{k} \eta\left(\mathbf{x}+\mathbf{d}_{\mathbf{i}}+\mathbf{m}\right) \eta\left(\mathbf{x}+\mathbf{c}+\mathbf{d}_{\mathbf{i}}+\mathbf{m}\right) .
\end{aligned}
$$

Notice that the set $\mathrm{A}+\mathrm{B}$ is also a box-lattice, denote it by $D \subseteq I_{N}^{n}$ and $B \cap(B-\mathbf{c})$ is a shifted version of a box lattice. The reason of this is that $B \cap(B-\mathbf{c})$ is non-empty only if $\mathbf{c}$ is a vector whose $i$-th coordinate is divisible by $z_{i}$, so $\mathbf{c}$ is of the form $\mathbf{c}=\left(c_{1} z_{1}, c_{2} z_{2}, \ldots, c_{n} z_{n}\right)$. Define $s_{i}$ by

$$
s_{i}= \begin{cases}0 & \text { if } c_{i} \geq 0 \\ -c_{i} z_{i} & \text { if } c_{i}<0\end{cases}
$$


and let $\mathbf{s}(c)$ be the vector $\mathbf{s}(c)=\left(s_{1}, s_{2}, \ldots, s_{n}\right)$. We define $B(c)$ as the following box lattice:

$$
\begin{gathered}
B(c)=\left\{\mathbf{x}=x_{1} \mathbf{u}_{\mathbf{1}}+\cdots+x_{n} \mathbf{u}_{\mathbf{n}}: 0 \leq x_{i} z_{i} \leq t_{i}-c_{i} z_{i}-s_{i} z_{i}(<N)\right. \\
\quad \text { for } i=1, \ldots, n\} .
\end{gathered}
$$

After introducing these notation it is not very difficult to see that $B \cap(B-\mathbf{c})$ is indeed a shifted box-lattice, namely

$$
B \cap(B-\mathbf{c})=\mathbf{s}(\mathbf{c})+B(c) .
$$

Moreover $A+B(c)$ is also a box-lattice, denote it by $D(c)$. Using these new notation we get

$$
\begin{aligned}
S= & \sum_{z \in D}\left(\prod_{i=1}^{k} \eta\left(\mathbf{z}+\mathbf{d}_{\mathbf{i}}\right)\right)^{2}+ \\
& +\sum_{\substack{\mathbf{c} \in C \\
\mathbf{c} \neq \mathbf{0}}} \sum_{\mathbf{z} \in D(c)} \prod_{i=1}^{k} \eta\left(\mathbf{z}+\mathbf{s}(\mathbf{c})+\mathbf{d}_{\mathbf{i}}\right) \eta\left(\mathbf{z}+\mathbf{s}(\mathbf{c})+\mathbf{c}+\mathbf{d}_{\mathbf{i}}\right) \\
\leq N^{n}+ & \sum_{\substack{\mathbf{c} \in C \\
\mathbf{c} \neq \mathbf{0}}}\left|\sum_{\mathbf{z} \in D(c)} \prod_{i=1}^{k} \eta\left(\mathbf{z}+\mathbf{s}(\mathbf{c})+\mathbf{d}_{\mathbf{i}}\right) \eta\left(\mathbf{z}+\mathbf{s}(\mathbf{c})+\mathbf{c}+\mathbf{d}_{\mathbf{i}}\right)\right|
\end{aligned}
$$

Next we estimate $\left|\sum_{\mathbf{z} \in D(c)} \prod_{i=1}^{k} \eta\left(\mathbf{z}+\mathbf{s}(\mathbf{c})+\mathbf{d}_{\mathbf{i}}\right) \eta\left(\mathbf{z}+\mathbf{s}(\mathbf{c})+\mathbf{c}+\mathbf{d}_{\mathbf{i}}\right)\right|$ by $Q_{2 k}(\eta)$ if the vectors $\mathbf{d}_{\mathbf{1}}, \mathbf{d}_{\mathbf{2}}, \ldots, \mathbf{d}_{\mathbf{k}}, \mathbf{c}+\mathbf{d}_{\mathbf{1}}, \mathbf{c}+\mathbf{d}_{\mathbf{2}}, \ldots, \mathbf{c}+\mathbf{d}_{\mathbf{k}}$ are all different. In the other case, when there are $i$ and $j$ for which $\mathbf{c}+\mathbf{d}_{\mathbf{i}}=\mathbf{d}_{\mathbf{j}}$, we will use the trivial estimate $N^{n}$. For every fixed $i$ and $j$ at most one $\mathbf{c}$ exists with $\mathbf{c}+\mathbf{d}_{\mathbf{i}}=\mathbf{d}_{\mathbf{j}}$, so we will use the trivial estimate only at most $k(k-1)$ times. Thus

$$
S \leq N^{n}+|C| Q_{2 k}(\eta)+k(k-1) N^{n} .
$$

Since $|C| \leq 2^{n} N^{n}$ and $k(k-1)+1 \leq k^{2}$ we get

$$
S \leq\left(2^{n}+k^{2}\right) Q_{2 k}(\eta) N^{n},
$$

which was to be proved. 


\section{Proof of Theorem 2}

In order to prove Theorem 2 we will give a construction for which

$$
N^{c n} \gg Q_{k}(\eta) \gg \sqrt{N^{n} C_{2 k}(\eta)} \gg N^{c n}
$$

holds. In our construction $N$ will always be a prime, thus we change our notation, and we write $p$ in place of $N$ (primes usually are denoted by $p$ ). The construction will be based on finite fields and their generators. Namely, let $\mathbb{F}_{p^{n}}$ be a finite field with $p^{n}$ elements, and let $g$ be a generator element of $\mathbb{F}_{p^{n}}^{*}\left(=\mathbb{F}_{p^{n}} \backslash\{0\}\right)$. Moreover, for $a \in \mathbb{F}_{p^{n}}^{*}$ define ind $a \in \mathbb{N}$ by

$$
g^{\text {ind } a}=a \quad \text { and } \quad 0 \leq \text { ind } a<p^{n}-1 .
$$

Let $v_{1}, v_{2}, \ldots, v_{n}$ be a basis of the vector space $\mathbb{F}_{p^{n}}$ over $\mathbb{F}_{p}$. We define the binary lattice $\eta: I_{p}^{n} \rightarrow\{-1,+1\}$ by

$$
\eta\left(x_{1}, x_{2}, \ldots, x_{n}\right)= \begin{cases}1 & \text { if } 0 \leq \text { ind }\left(x_{1} v_{1}+x_{2} v_{2}+\cdots+x_{n} v_{n}\right) \leq L-1 \\ -1 & \text { if } L \leq \text { ind }\left(x_{1} v_{1}+x_{2} v_{2}+\cdots+x_{n} v_{n}\right)<p^{n}-1 \\ & \text { or }\left(x_{1}, x_{2}, \ldots, x_{n}\right)=(0,0, \ldots, 0)\end{cases}
$$

where $L$ is a positive integer with $1 \leq L \leq p^{n}-1$. The exact value of $L$ will be defined later. We claim that for optimally chosen $L$ we have

$$
p^{c n} \gg Q_{k}(\eta) \gg \sqrt{p^{n} C_{2 k}(\eta)} \gg p^{c n},
$$

which proves the theorem. In order to estimate $Q_{k}(\eta)$ and $C_{2 k}(\eta)$ we need to prove the following lemma:

Lemma 3. Consider the binary lattice $\eta$ defined by (3.1) where $L$ is a positive integer with $1 \leq L \leq p^{n}-1$. Define $S$ by $S \stackrel{\text { def }}{=} L-\frac{p^{n}-1}{2}$. let $B$ be a box $N$-lattice. Then

$$
\begin{aligned}
& \left|\sum_{\mathbf{x} \in B} \eta\left(\mathbf{x}+\mathbf{d}_{1}\right) \eta\left(\mathbf{x}+\mathbf{d}_{2}\right) \cdots \eta\left(\mathbf{x}+\mathbf{d}_{\ell}\right)\right| \\
& =\frac{2^{\ell}}{\left(p^{n}-1\right)^{\ell}} S^{\ell}|B|+O\left(\ell(4 n)^{\ell} \sqrt{p^{n}}(1+\log p)^{n+\ell}\right) .
\end{aligned}
$$


In order to handle the sum in (3.2) we will use characters over $\mathbb{F}_{p^{n}}$. First, we express $\eta(\mathbf{x})$ by character sums. We will use the formula

$$
\frac{1}{p^{n}-1} \sum_{\chi} \bar{\chi}(a) \chi(b)=\left\{\begin{array}{ll}
1 & \text { if } a=b \\
0 & \text { if } a \neq b
\end{array},\right.
$$

where the sum runs over all multiplicative characters $\chi$ over $\mathbb{F}_{p^{n}}$. By this formula for $\mathbf{x} \neq \mathbf{0}$ we have

$$
\begin{aligned}
\eta(\mathbf{x}) & =2 \quad \sum_{\substack{0 \leq j \leq L-1 \\
j=\text { ind } \\
\left(x_{1} v_{1}+\cdots+x_{n} v_{n}\right)}} 1-1= \\
& =\frac{2}{p^{n}-1} \sum_{0 \leq j \leq L-1} \sum_{\chi} \bar{\chi}\left(x_{1} v_{1}+\cdots+x_{n} v_{n}\right) \chi\left(g^{j}\right)-1 \\
& =\frac{2}{p^{n}-1} \sum_{0 \leq j \leq L-1} \sum_{\chi \neq \chi_{0}} \bar{\chi}\left(x_{1} v_{1}+\cdots+x_{n} v_{n}\right) \chi\left(g^{j}\right)+\frac{2 S}{p^{n}-1}
\end{aligned}
$$

We would like to estimate sums of form $\left|\sum_{\mathbf{x} \in B} \eta\left(\mathbf{x}+\mathbf{d}_{\mathbf{1}}\right) \cdots \eta\left(\mathbf{x}+\mathbf{d}_{\ell}\right)\right|$. Write $\mathbf{d}_{\mathbf{i}}=\left(d_{i}^{(1)}, d_{i}^{(2)}, \ldots, d_{i}^{(n)}\right)$. Then for $\mathbf{x} \neq \mathbf{0}$ we have

$$
\begin{aligned}
& \eta\left(\mathbf{x}+\mathbf{d}_{1}\right) \eta\left(\mathbf{x}+\mathbf{d}_{2}\right) \cdots \eta\left(\mathbf{x}+\mathbf{d}_{\ell}\right)=\frac{2^{\ell}}{\left(p^{n}-1\right)^{\ell}} . \\
& \cdot \prod_{i=1}^{\ell}\left(\sum_{j=0}^{L-1} \sum_{\chi \neq \chi_{0}} \bar{\chi}\left(v_{1}\left(x_{1}+d_{i}^{(1)}\right)+\cdots+v_{n}\left(x_{n}+d_{i}^{(n)}\right)\right) \chi\left(g^{j}\right)+S\right) \\
& =\frac{2^{\ell}}{\left(p^{n}-1\right)^{\ell}} \sum_{\left\{i_{1}, i_{2} \ldots, i_{t}\right\} \subset\{1,2, \ldots, \ell\}} S^{\ell-t} \sum_{\chi_{i_{1}} \neq \chi_{0}} \cdots \sum_{\chi_{i_{t}} \neq \chi_{0}} \\
& \overline{\chi_{i_{1}}}\left(v_{1}\left(x_{1}+d_{i_{1}}^{(1)}\right)+\cdots+v_{n}\left(x_{n}+d_{i_{1}}^{(n)}\right)\right) \cdots \\
& \overline{\chi_{i_{t}}}\left(v_{1}\left(x_{1}+d_{i_{t}}^{(1)}\right)+\cdots+v_{n}\left(x_{n}+d_{i_{t}}^{(n)}\right)\right) \times \prod_{j=1}^{t}\left(\sum_{r=0}^{L-1} \chi_{i_{j}}\left(g^{r}\right)\right) .
\end{aligned}
$$

Here in the first sum of the right-hand side of the inequality we write the 
term $t=0$ separately:

$$
\begin{aligned}
& \eta\left(\mathbf{x}+\mathbf{d}_{1}\right) \eta\left(\mathbf{x}+\mathbf{d}_{2}\right) \cdots \eta\left(\mathbf{x}+\mathbf{d}_{\ell}\right)=\frac{2^{\ell}}{\left(p^{n}-1\right)^{\ell}} S^{\ell}+ \\
& +\frac{2^{\ell}}{\left(p^{n}-1\right)^{\ell}} \sum_{\substack{\left.i_{1}, i_{2} \ldots, i_{t}\right\} \subset\{1,2, \ldots, \ell\} \\
1 \leq t \leq \ell}} S^{\ell-t} \sum_{\substack{\chi_{i_{1}} \neq \chi_{0} \\
\chi_{i_{t}} \neq \chi_{0}}} \cdots \sum_{\overline{\chi_{i_{1}}}}\left(v_{1}\left(x_{1}+d_{i_{1}}^{(1)}\right)+\cdots+v_{n}\left(x_{n}+d_{i_{1}}^{(n)}\right)\right) \cdots \\
& \overline{\chi_{i_{t}}}\left(v_{1}\left(x_{1}+d_{i_{t}}^{(1)}\right)+\cdots+v_{n}\left(x_{n}+d_{i_{t}}^{(n)}\right)\right) \times \prod_{j=1}^{t}\left(\sum_{r=0}^{L-1} \chi_{i_{j}}\left(g^{r}\right)\right) .
\end{aligned}
$$

Next we consider the sum of those terms where $\mathbf{x} \in B$ :

$$
\begin{aligned}
& \sum_{\mathbf{x} \in B} \eta\left(\mathbf{x}+\mathbf{d}_{1}\right) \eta\left(\mathbf{x}+\mathbf{d}_{2}\right) \cdots \eta\left(\mathbf{x}+\mathbf{d}_{\ell}\right)= \\
& =\frac{2^{\ell}}{\left(p^{n}-1\right)^{\ell}} S^{\ell}|B|+\frac{2^{\ell}}{\left(p^{n}-1\right)^{\ell}} \sum_{\substack{\left\{i_{1}, i_{2} \ldots, i_{t}\right\} \subset\{1,2, \ldots, \ell\} \\
1 \leq t \leq \ell}} S^{\ell-t} \sum_{\substack{\chi_{i_{1}} \neq \chi_{0} \\
\chi_{i_{t}} \neq \chi_{0}}} \\
& \left(\sum_{\mathbf{x} \in B} \overline{\chi_{i_{1}}}\left(v_{1}\left(x_{1}+d_{i}^{(1)}\right)+\cdots+v_{n}\left(x_{n}+d_{i}^{(n)}\right)\right) \cdots\right. \\
& \left.\overline{\chi_{i_{t}}}\left(v_{1}\left(x_{1}+d_{i}^{(1)}\right)+\cdots+v_{n}\left(x_{n}+d_{i}^{(n)}\right)\right)\right) \times \prod_{j=1}^{t}\left(\sum_{r=0}^{L-1} \chi_{i_{j}}\left(g^{r}\right)\right) .
\end{aligned}
$$

Using the triangle inequality we get that there exists a $-1 \leq \varepsilon \leq 1$ such that

$$
\begin{aligned}
& \sum_{\mathbf{x} \in B} \eta\left(\mathbf{x}+\mathbf{d}_{1}\right) \eta\left(\mathbf{x}+\mathbf{d}_{2}\right) \cdots \eta\left(\mathbf{x}+\mathbf{d}_{\ell}\right)= \\
& =\frac{2^{\ell}}{\left(p^{n}-1\right)^{\ell}} S^{\ell}|B|+\varepsilon \frac{2^{\ell}}{\left(p^{n}-1\right)^{\ell}} \sum_{\substack{\left\{i_{1}, i_{2} \ldots, i_{t}\right\} \subset\{1,2, \ldots, \ell\} \\
1 \leq t \leq \ell}} S^{\ell-t} \sum_{\substack{\chi_{i_{1}} \neq \chi_{0} \\
\chi_{i_{t}} \neq \chi_{0}}} \cdots \sum_{\mathbf{x} \in B} \overline{\chi_{i_{1}}}\left(v_{1}\left(x_{1}+d_{i_{1}}^{(1)}\right)+\cdots+v_{n}\left(x_{n}+d_{i_{1}}^{(n)}\right)\right) \cdots \\
& \overline{\chi_{i_{t}}}\left(v_{1}\left(x_{1}+d_{i_{t}}^{(1)}\right)+\cdots+v_{n}\left(x_{n}+d_{i_{t}}^{(n)}\right)\right)|\times| \prod_{j=1}^{t}\left(\sum_{r=0}^{L-1} \chi_{i_{j}}\left(g^{r}\right)\right) \mid .
\end{aligned}
$$


The characters over $\mathbb{F}_{q}$ form a cyclic group, whose generator element will be denoted by $\chi_{1}$. Fix $i_{1}, i_{2}, \ldots, i_{t}$ and consider the sum

$$
\begin{aligned}
& \mid \sum_{\mathbf{x} \in B} \overline{\chi_{i_{1}}}\left(v_{1}\left(x_{1}+d_{i_{1}}^{(1)}\right)+\cdots+v_{n}\left(x_{n}+d_{i_{1}}^{(n)}\right)\right) \cdots \\
& \overline{\chi_{i_{t}}}\left(v_{1}\left(x_{1}+d_{i_{t}}^{(1)}\right)+\cdots+v_{n}\left(x_{n}+d_{i_{t}}^{(n)}\right)\right) \mid
\end{aligned}
$$

in (3.3). Here $\chi_{i_{j}}$ is in the form $\chi_{i_{j}}=\chi_{1}^{\alpha_{j}}$ where $(q-1) \nmid \alpha_{j}$. Moreover write

$$
\begin{aligned}
& g\left(x_{1} v_{1}+\cdots+x_{n} v_{n}\right) \stackrel{\text { def }}{=}\left(x_{1} v_{1}+\cdots+x_{n} v_{n}+v_{1} d_{i_{1}}^{(1)}+\ldots v_{n} d_{i_{1}}^{(n)}\right) \\
& \cdot\left(x_{1} v_{1}+\cdots+x_{n} v_{n}+v_{1} d_{i_{2}}^{(1)}+\ldots v_{n} d_{i_{2}}^{(n)}\right) \\
& \vdots \\
& \cdot\left(x_{1} v_{1}+\cdots+x_{n} v_{n}+v_{1} d_{i_{t}}^{(1)}+\ldots v_{n} d_{i_{t}}^{(n)}\right)
\end{aligned}
$$

Then

$$
\begin{aligned}
& \overline{\chi_{i_{1}}}\left(v_{1}\left(x_{1}+d_{i_{1}}^{(1)}\right)+\cdots+v_{n}\left(x_{n}+d_{i_{1}}^{(n)}\right)\right) \cdots \\
& \overline{\chi_{i_{t}}}\left(v_{1}\left(x_{1}+d_{i_{t}}^{(1)}\right)+\cdots+v_{n}\left(x_{n}+d_{i_{t}}^{(n)}\right)\right) \\
& =\overline{\chi_{1}}\left(\left(v_{1}\left(x_{1}+d_{i_{1}}^{(1)}\right)+\cdots+v_{n}\left(x_{n}+d_{i_{1}}^{(n)}\right)\right)^{\alpha_{1}} \ldots\right. \\
& \left.\left(v_{1}\left(x_{1}+d_{i_{t}}^{(1)}\right)+\cdots+v_{n}\left(x_{n}+d_{i_{t}}^{(n)}\right)\right)^{\alpha_{t}}\right) \\
& =\overline{\chi_{1}}\left(\left(x_{1} v_{1}+\cdots+x_{n} v_{n}+v_{1} d_{i_{1}}^{(1)}+\ldots v_{n} d_{i_{1}}^{(n)}\right)^{\alpha_{1}} \ldots\right. \\
& \left.\left(x_{1} v_{1}+\cdots+x_{n} v_{n}+v_{1} d_{i_{t}}^{(1)}+\ldots v_{n} d_{i_{t}}^{(n)}\right)^{\alpha_{t}}\right) \\
& =\overline{\chi_{1}}\left(g\left(x_{1} v_{x}+\cdots+x_{n} v_{n}\right)\right) .
\end{aligned}
$$


By this we get

$$
\begin{aligned}
& \mid \sum_{\mathbf{x} \in B} \overline{\chi_{i_{1}}}\left(v_{1}\left(x_{1}+d_{i_{1}}^{(1)}\right)+\cdots+v_{n}\left(x_{n}+d_{i_{1}}^{(n)}\right)\right) \cdots \\
& \overline{\chi_{i_{t}}}\left(v_{1}\left(x_{1}+d_{i_{t}}^{(1)}\right)+\cdots+v_{n}\left(x_{n}+d_{i_{t}}^{(n)}\right)\right) \mid \\
& =\left|\sum_{\mathbf{x} \in B} \overline{\chi_{1}}\left(g\left(x_{1} v_{1}+\cdots+x_{n} v_{n}\right)\right)\right| .
\end{aligned}
$$

Winterhof proved in [13] the following lemma:

Lemma 4. Suppose that $\chi$ is a non-trivial multiplicative character of order d, and $f(x)$ is a polynomial which is not of the form $c g(x)^{d}$, where $g(x) \in \mathbb{F}_{q}[x]$ and $f(x)$ has $m$ distinct zeros in its splitting field $\mathbb{F}_{q}$. Then for $1 \leq k_{i} \leq p$; $i=1, \ldots, n$ let

$$
B=B\left(k_{1}, k_{2}, \ldots, k_{n}\right)=\left\{x_{1} v_{1}+\cdots+x_{n} v_{n}: 0 \leq x_{i}<k_{i}, i=1,2, \ldots, n\right\}
$$

Then for any $1 \leq k_{i} \leq p ; i=1,2, \ldots, n$ we have

$$
\left|\sum_{z \in B} \chi(f(z))\right|<m q^{1 / 2}(1+\log p)^{n} .
$$

By Lemma 4 and (3.4) we get

$$
\begin{aligned}
& \mid \sum_{\mathbf{x} \in B} \overline{\chi_{i_{1}}}\left(v_{1}\left(x_{1}+d_{i_{1}}^{(1)}\right)+\cdots+v_{n}\left(x_{n}+d_{i_{1}}^{(n)}\right)\right) \cdots \\
& \overline{\chi_{i_{t}}}\left(v_{1}\left(x_{1}+d_{i_{t}}^{(1)}\right)+\cdots+v_{n}\left(x_{n}+d_{i_{t}}^{(n)}\right)\right) \mid \\
& =\left|\sum_{\mathbf{x} \in B} \overline{\chi_{1}}\left(g\left(x_{1} v_{1}+\cdots+x_{n} v_{n}\right)\right)\right| \\
& \leq \ell \sqrt{p^{n}}(1+\log p)^{n} .
\end{aligned}
$$


Thus

$$
\begin{aligned}
& \sum_{\mathbf{x} \in B} \eta\left(\mathbf{x}+\mathbf{d}_{1}\right) \eta\left(\mathbf{x}+\mathbf{d}_{2}\right) \cdots \eta\left(\mathbf{x}+\mathbf{d}_{\ell}\right)= \\
& =\frac{2^{\ell}}{\left(p^{n}-1\right)^{\ell}} S^{\ell}|B|+O\left(\frac{2^{\ell}}{\left(p^{n}-1\right)^{\ell}} \sum_{\substack{\left\{i_{1}, i_{2} \ldots, i_{t}\right\} \subset\{1,2, \ldots, \ell\} \\
1 \leq t \leq \ell}} S^{\ell-t} \sum_{\substack{1 \leq t, \ell \\
\chi_{1} \neq \chi_{0}}} \cdots \sum_{\chi_{i_{t}} \neq \chi_{0}}\right. \\
& \left.\ell \sqrt{p^{n}}(1+\log p)^{n} \times\left|\prod_{j=1}^{t}\left(\sum_{r=0}^{L-1} \chi_{i_{j}}\left(g^{r}\right)\right)\right|\right)
\end{aligned}
$$

Here $\left|\sum_{r=0}^{L-1} \chi_{i_{j}}\left(g^{r}\right)\right|=\frac{\left|1-\chi_{i_{j}}(g)^{L}\right|}{\left|1-\chi_{i_{j}}(g)\right|} \leq \frac{2}{\left|1-\chi_{i_{j}}(g)\right|}$ so

$$
\begin{aligned}
& \sum_{\mathbf{x} \in B} \eta\left(\mathbf{x}+\mathbf{d}_{1}\right) \eta\left(\mathbf{x}+\mathbf{d}_{2}\right) \cdots \eta\left(\mathbf{x}+\mathbf{d}_{\ell}\right)=\frac{2^{\ell}}{\left(p^{n}-1\right)^{\ell}} S^{\ell}|B|+ \\
& +O\left(\frac{\ell 2^{\ell} \sqrt{p^{n}}(1+\log p)^{n}}{\left(p^{n}-1\right)^{\ell}} \sum_{\substack{\left\{i_{1}, \ldots, i_{t}\right\} \subset\{1,2, \ldots, \ell\} \\
1 \leq t \leq \ell}} S^{\ell-t} \sum_{\chi_{i_{1}} \neq \chi_{0}} \cdots \sum_{\chi_{i_{t}} \neq \chi_{0}}\right. \\
& \left.\prod_{j=1}^{t} \frac{2}{\left|1-\chi_{i_{j}}(g)\right|}\right) .
\end{aligned}
$$

Thus

$$
\begin{aligned}
& \sum_{\mathbf{x} \in B} \eta\left(\mathbf{x}+\mathbf{d}_{1}\right) \eta\left(\mathbf{x}+\mathbf{d}_{2}\right) \cdots \eta\left(\mathbf{x}+\mathbf{d}_{\ell}\right)= \\
& =\frac{2^{\ell}}{\left(p^{n}-1\right)^{\ell}} S^{\ell}|B|+O\left(\frac{\ell 2^{\ell} \sqrt{p^{n}}(1+\log p)^{n}}{\left(p^{n}-1\right)^{\ell}}\left(S+\sum_{\chi \neq \chi_{0}} \frac{2}{|1-\chi(g)|}\right)^{\ell}\right) .
\end{aligned}
$$

Now $\chi_{1}$ is a generator of the group of characters over $\mathbb{F}_{q}$. More precisely, since 
$g$ is a generator element of $\mathbb{F}_{p^{n}}^{*}$, we may define $\chi_{1}$ by $\chi_{1}(g)=e^{2 \pi i /\left(p^{n}-1\right)}$. Then

$$
\begin{aligned}
\sum_{\chi \neq \chi_{0}} \frac{1}{|1-\chi(g)|} & =\sum_{j=1}^{p^{n}-2} \frac{1}{\left|1-\chi^{j}(g)\right|}=\sum_{j=1}^{p^{n}-2} \frac{1}{\mid 1-e^{2 \pi i j /\left(p^{n}-1\right) \mid}} \\
& \leq \frac{1}{4} \sum_{j=1}^{p^{n}-2} \frac{1}{\| j /\left(p^{n}-1\right) \mid} \leq \frac{1}{2} \sum_{j=1}^{\left(p^{n}-1\right) / 2} \frac{1}{\left\|j /\left(p^{n}-1\right)\right\|} \\
& =\frac{1}{2} \sum_{j=1}^{\left(p^{n}-1\right) / 2} \frac{p^{n}-1}{j}<\frac{1}{2}\left(p^{n}-1\right)\left(1+\log \left(p^{n} / 2\right)\right) \\
& <n\left(p^{n}-1\right) \log p^{n} .
\end{aligned}
$$

Thus

$$
\begin{aligned}
& \sum_{\mathbf{x} \in B} \eta\left(\mathbf{x}+\mathbf{d}_{1}\right) \eta\left(\mathbf{x}+\mathbf{d}_{2}\right) \cdots \eta\left(\mathbf{x}+\mathbf{d}_{\ell}\right)= \\
& =\frac{2^{\ell}}{\left(p^{n}-1\right)^{\ell}} S^{\ell}|B|+O\left(\frac{\ell 2^{\ell} \sqrt{p^{n}}(1+\log p)^{n}}{\left(p^{n}-1\right)^{\ell}}\left(S+n\left(p^{n}-1\right) \log p\right)^{\ell}\right) .
\end{aligned}
$$

Now we fix the value of $L$ as $L=\frac{p^{n}-1}{2}+\left[p^{1-(1-c) / n}\right]$ so that $S=\left[p^{1-(1-c) / n}\right]$. Then $S<n\left(p^{n}-1\right) \log p$, thus

$$
\begin{aligned}
& \sum_{\mathbf{x} \in B} \eta\left(\mathbf{x}+\mathbf{d}_{1}\right) \eta\left(\mathbf{x}+\mathbf{d}_{2}\right) \cdots \eta\left(\mathbf{x}+\mathbf{d}_{\ell}\right)= \\
& =\frac{2^{\ell}}{\left(p^{n}-1\right)^{\ell}} S^{\ell}|B|+O\left(\frac{\ell 2^{\ell} \sqrt{p^{n}}(1+\log p)^{n}}{\left(p^{n}-1\right)^{\ell}}\left(2 n\left(p^{n}-1\right) \log p\right)^{\ell}\right) \\
& =\frac{2^{\ell}}{\left(p^{n}-1\right)^{\ell}} S^{\ell}|B|+O\left(\ell(4 n)^{\ell} \sqrt{p^{n}}(1+\log p)^{n+\ell}\right) .
\end{aligned}
$$

The maximum value of $|B|$ is $p^{n}-1$, thus

$$
\begin{aligned}
Q_{k}(\eta) & =\frac{2^{k}}{\left(p^{n}-1\right)^{k-1}} S^{k}+O\left(k \cdot(4 n)^{k} \sqrt{p^{n}}(1+\log p)^{n+k}\right) \\
C_{2 k}(\eta) & =\frac{2^{2 k}}{\left(p^{n}-1\right)^{2 k-1}} S^{2 k}+O\left(2 k \cdot(4 n)^{2 k} \sqrt{p^{n}}(1+\log p)^{n+2 k}\right) .
\end{aligned}
$$


Using $\frac{S^{k}}{\left(p^{n}-1\right)^{k}}>O\left(k \cdot(4 n)^{k} \sqrt{p^{n}}(1+\log p)^{n+k}\right)$

and $\frac{S^{2 k}}{\left(p^{n}-1\right)^{2 k}}>O\left(2 k \cdot(4 n)^{2 k} \sqrt{p^{n}}(1+\log p)^{n+2 k}\right)$ if $c>3 / 4$ and $p$ is large enough, we get

$$
\begin{aligned}
Q_{k}(\eta) & \leq \frac{2^{k}+1}{\left(p^{n}-1\right)^{k-1}} S^{k} \\
C_{2 k}(\eta) & \geq \frac{2^{2 k}-1}{\left(p^{n}-1\right)^{2 k-1}} S^{2 k} .
\end{aligned}
$$

By $S=\left[p^{1-(1-c) / k}\right]$ we obtain

$$
p^{c n} \gg Q_{k}(\eta) \gg \sqrt{p^{n} C_{2 k}(\eta)} \gg p^{c n}
$$

which was to be proved.

\section{References}

[1] N. Alon, Y. Kohayakawa, C. Mauduit, C. G. Moreira and V. Rödl, Measures of pseudorandomness for finite sequences: minimal values, Combin., Probab. Comput. 15 (2005), 1-29.

[2] J. Cassaigne, C. Mauduit and A. Sárközy, On finite pseudorandom binary sequences VII: The measures of pseudorandomness, Acta Arith. 103 (2002), 97-118.

[3] K. Gyarmati, An inequality between the measures of pseudorandomness, Ann. Univ. Sci. Budapest. Eötvös Sect. Math. 46 (2003), 157-166.

[4] K. Gyarmati, Measures of pseudorandomness, P. Charpin, A. Pott, A. Winterhof (eds.), Radon Series in Computational and Applied Mathematics, de Gruyter (2013), 43-64.

[5] K. Gyarmati, C. Mauduit, A. Sárközy, Measures of pseudorandomness of finite binary lattices, III ( $Q_{k}$, correlation, normality, minimal values.), Unif. Distrib. Theory 5 (2010), 183-207. 
[6] K. Gyarmati, A. Sárközy and C. Stewart, On Legendre symbol lattices, Unif. Distri. Theory 4 (2009), no. 1, 81-95.

[7] P. Hubert, C. Mauduit and A. Sárközy On pseudorandom binary lattices, Acta Arith. 125 (2006), 51-62.

[8] C. Mauduit and A. Sárközy, On finite pseudorandom binary sequences I: Measures of pseudorandomness, the Legendre symbol, Acta Arith. 82 (1997), 365-377.

[9] C. Mauduit and A. Sárközy, On the measures of pseudorandomness of binary sequences, Discrete Math. 271 (2003), 195-207.

[10] A. Sárközy, On pseudorandomness of families of binary sequences, Discrete Applied Math. 216 (2017), 670-676.

[11] R. Sebók, On a connection between pseudorandom measures, Unif. Distrib. Theory 10 (2015), 107-113.

[12] A. Weil, Sur les courbes algébriques et les variétés qui s'en déduisent, Act. Sci. Ind. 1041, Hermann, Paris, (1948).

[13] A. Winterhof, Some estimates for character sums and applications, Des. Codes and Cryptogr. 22, (2001). 123-131. 